\title{
ON MATRICES WHOSE COEFFICIENTS ARE FUNCTIONS OF A SINGLE VARIABLE
}

BY

\section{J. H. M. WEDDERBURN}

1. The methods usually* employed in reducing to its normal form a matrix whose coefficients are polynomials in a variable $\lambda$ are of such a nature that it is not at all obvious how they can be extended when polynomials are replaced by analytic functions. $\dagger$. The object of this note is to show that the main theorems on elementary factors are not restricted to matric polynomials but apply without appreciable modification to analytic matric functions.

As vectors are employed freely in the sequel, a short explanation of the notation used is necessary. A vector, $x=\left(\xi_{1}, \xi_{2}, \cdots, \xi_{n}\right)$, is an ordered set of $n$ coefficients: two vectors are equal if, and only if, their corresponding coefficients are equal. The sum of two vectors, $x=\left(\xi_{1}, \xi_{2}, \cdots, \xi_{n}\right)$ and $y=\left(\eta_{1}, \eta_{2}, \cdots, \eta_{n}\right)$ is defined as

$$
x+y=\left(\xi_{1}+\eta_{1}, \xi_{2}+\eta_{2}, \cdots, \xi_{n}+\eta_{n}\right) .
$$

The product of any number $\rho$ into a vector $x$ is defined as

$$
\rho x=\left(\rho \xi_{1}, \rho \xi_{2}, \cdots, \rho \xi_{n}\right) .
$$

Except in this elementary case, multiplication of vectors will not be used. A set of $n$ linearly independent vectors is called a basis. Any vector can be expressed linearly in terms of the elements of a basis and, in particular, if $e_{i}$ is the vector for which $\xi_{i}=1, \xi_{j}=0(j \neq i)$, we have $x=\Sigma_{1}^{n} \xi_{i} e_{i}$.

If $A$ is a matrix $\left(a_{r s}\right)$ and $x=\Sigma \xi_{i} e_{i}$, the vector $\Sigma_{i=1}^{i=n}\left(\sum_{j=1}^{j=n} a_{i j} \xi_{j}\right) e_{i}$ is denoted by $A x$. We evidently have

$A\left(x_{1}+x_{2}\right)=A x_{1}+A x_{2}, \quad(A+B) x=A x+B x, \quad A \cdot B x=(A B) x$.

A matrix is completely determined when its action on $n$ linearly independent vectors is given. If the determinant of a matrix is not zero, it transforms a set of linearly independent vectors into a linearly independent set; while if its rank is $r(r<n)$, there is a unique complex of vectors every element of

* See for instance Bôcher, Introduction to Higher Algebra, p. 262.

$\dagger$ To avoid frequent repetition, a matrix whose coefficients are functions of a variable, $\lambda$, will be called a matric function of $\lambda$. 
which is annihilated by the matrix. The following lemma is an immediate consequence of this.

LemmA I. If $x_{1}, x_{2}, \cdots, x_{n}$ is a set of vector functions which are holomorphic and linearly independent for all values of $\lambda$ lying in a given region $R$, the determinant of the matrix defined by

$$
x_{r}=P(\lambda) e_{r} \quad(r=1,2, \cdots, n)
$$

does not vanish for any value of $\lambda$ in $R$.

It may be remarked that the matrix $P(\lambda)$ is holomorphic in $R$ and, since $|P(\lambda)|^{*}$ does not vanish, $P^{-1}(\lambda)$ is also holomorphic in that region. Also, since

$$
e_{r}=P^{-1}(\lambda) x_{r}, \quad(r=1,2, \cdots, n),
$$

the fixed basis $e_{1}, e_{2}, \cdots, e_{n}$ may be replaced by any set of vector functions which are holomorphic and linearly independent throughout $R$.

2. In the reduction of a matrix to its normal form, we require the following extension of a well-known algebraic theorem.

Lemma II. If $f(\lambda)$ and $g(\lambda)$ are two functions which are holomorphic in $R$ and have no factor $\dagger$ in common in that region, there exist two functions, holomorphic in $R$, such that

$$
p(\lambda) f(\lambda)+q(\lambda) g(\lambda) \equiv 1 .
$$

If we expand $1 /[f(\lambda) g(\lambda)]$ in terms of its principal parts in a MittagLeffler series $\ddagger$ we get

$$
\frac{1}{f(\lambda) g(\lambda)}=F(\lambda)+G(\lambda)+\phi(\lambda)
$$

where $F(\lambda)$ and $G(\lambda)$ are the parts of the series arising from the zeros of $f(\lambda)$ and $g(\lambda)$, respectively, which lie in $R$, and $\phi(\lambda)$ is a function which is holomorphic in $R$. If we now put

$$
p(\lambda)=g(\lambda)[G(\lambda)+\phi(\lambda)], \quad q(\lambda)=f(\lambda) F(\lambda),
$$

both $p(\lambda)$ and $q(\lambda)$ are holomorphic in $R$ and, on multiplying both sides of (1) by $f(\lambda) g(\lambda)$, we have

$1=f(\lambda) \cdot g(\lambda)[G(\lambda)+\phi(\lambda)]+g(\lambda) \cdot f(\lambda) F(\lambda)$

as required by the Lemma.

$$
=f(\lambda) p(\lambda)+g(\lambda) q(\lambda),
$$

* The determinant of the matrix $P(\lambda)$ is denoted by $|P(\lambda)|$.

$\dagger$ I. e., the two functions have no common zeros in $R$. Functions which are holomorphic and nowhere zero in $R$ play the same rôle as constants do in the algebraic theory and will therefore not be regarded as factors in this paper.

¥ The series used here is a special case of the "generalized Mittag-Leffler theorem." See Osgood, Funktionentheorie vol. 1 (second edition), p. 540, or Mittag-Leffler, A c t a M a t h e m a t i c a, vol. 4 (1884), p. 8. 
3. Lemma III. If $p_{1}(\boldsymbol{\lambda}), p_{2}(\boldsymbol{\lambda}), \cdots, p_{n}(\boldsymbol{\lambda})$ are $n$ functions which are holomorphic and have no factor common to all in $R$, and $x_{1}, x_{2}, \cdots, x_{n}$ is a set of vector functions which are holomorphic and linearly independent for all values of $\lambda$ in $R$, there exists a matric function $P(\lambda)$, holomorphic in $R$, for which $(i)|P(\lambda)| \neq 0$ for any $\lambda$ in $R$, and (ii)

$$
P(\lambda) x_{1}=p_{1} x_{1}+p_{2} x_{2}+\cdots+p_{n} x_{n} .
$$

Assume that the lemma is true for bases of order less than $n$ : then, if $l$ is the H.C.F. of $p_{1}, p_{2}, \cdots, p_{n-1}$ and $p_{r}^{\prime}=p_{r} / l,(r=1,2, \cdots, n-1)$, there exists a matric function, $Q_{0}$, relative to the basis $x_{1}, x_{2}, \cdots, x_{n-1}$ which is holomorphic and never singular in $R$ and is such that

$$
Q_{0} x_{1}=p_{1}^{\prime} x_{1}+p_{2}^{\prime} x_{2}+\cdots+p_{n-1}^{\prime} x_{n-1} \text {. }
$$

Let $Q_{1}$ be the matrix defined relatively to the basis $x_{1}, x_{2}, \cdots, x_{n}$ by

$$
Q_{1} x_{n}=x_{n}, \quad Q_{1} x_{r}=Q_{0} x_{r} \quad(r=1,2, \cdots, n-1),
$$

and set $x_{r}^{\prime}=Q_{1} x_{r}(r=1,2, \cdots, n)$. Since $l$ and $p_{n}$ have no common factor in $R$, we can, as in Lemma II, find two functions, $\alpha$ and $\beta$, which are holomorphic in $R$ and are such that ${ }^{*} \alpha l+\beta p_{n} \equiv 1$. If therefore $Q_{2}$ is the matrix defined by

$$
\begin{array}{ll}
Q_{2} x_{1}^{\prime}=l x_{1}^{\prime}+p_{n} x_{n}^{\prime}, \quad Q_{2} x_{n}^{\prime}=-\beta x_{2}^{\prime}+\alpha x_{n}^{\prime}, \quad Q_{2} x_{r}^{\prime}=x_{r}^{\prime} \\
& (r=2, \cdots, n-1),
\end{array}
$$

we have $\left|Q_{2}\right|=1$, and $P(\lambda)=Q_{2} Q_{1}$ satisfies the conditions of the lemma since

$$
P x_{1}=Q_{2} Q_{1} x_{1}=Q_{2} x_{1}^{\prime}=l x_{1}^{\prime}+p_{n} x_{n}^{\prime}=\sum_{1}^{n} p_{r} x_{r} .
$$

Since the lemma is obviously true for $n=1$, the required result follows immediately by induction.

4. Theorem. If $A(\lambda)$ is a matric function of rank $r$ which is holomorphic in a region $R$, there exist two matric functions, $P(\lambda)$ and $Q(\lambda)$, which are holomorphic and non-singular in $R$, and are such that

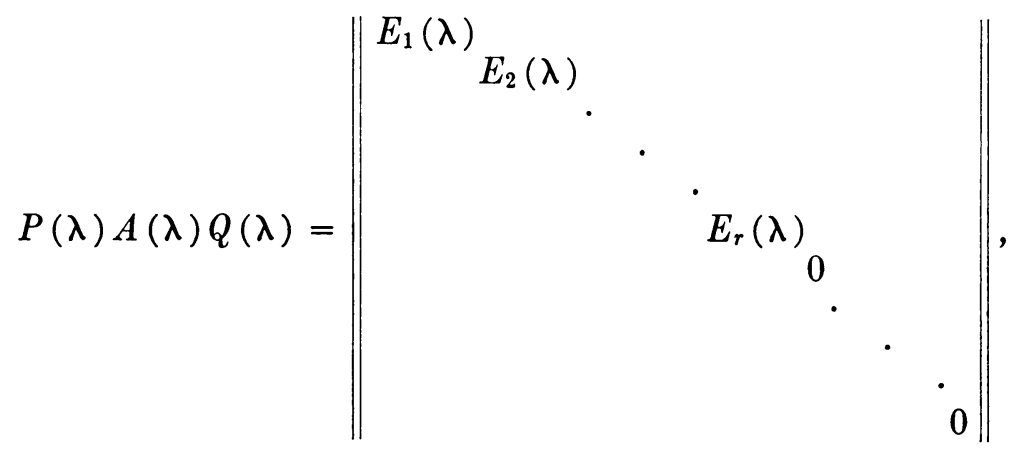

*If $p_{n}=0$, then $l=1=\alpha$. 
where $E_{1}(\lambda), \cdots E_{r}(\lambda)$ are functions of $\lambda$ which are holomorphic in $R$ and are such that $E_{s}$ is a factor of $E_{\iota}$ when $s<t(s, t=1,2, \cdots, r)$.

This theorem is obviously true when $n=1$ so that we may make its proof depend on induction. We assume therefore that it is true for matrices of order less than $n$.

If the rank of $A$ is less than $n$, there is at least one vector function $x$, holomorphic in $R$, for which $A x \equiv 0$ in $R$; and we can insure that $x$ itself does not vanish by removing any common factor from its coefficients when expressed in terms of $e_{1}, e_{2}, \cdots, e_{n}$. By Lemma III, there is a matric function, $Q_{0}$, holomorphic and non-singular in $R$, for which $x=Q_{0} e_{n}$, whence $A Q_{0} e_{n} \equiv 0$ : the elements in the last column of $A Q_{0}$ are therefore all zero. Considering now the conjugate matrix $Q_{0}^{\prime} A^{\prime}$, we see in the same manner that there is a matric function $P_{0}^{\prime}$ holomorphic and non-singular in $R$, which is such that the coefficients in the last column of $Q_{0}^{\prime} A^{\prime}$ are also all zero. It follows then that $P_{0} A Q_{0}$ has zeros both in the last column and in the last row, and may therefore be regarded as a matrix of order $n-1$ relative to the basis $e_{1}, e_{2}, \cdots, e_{n-1}$. There are therefore by hypothesis two matric functions, $P_{1}$ and $Q_{1}$, of order $n-1$ which are holomorphic and non-singular in $R$ and are such that $P_{1} P_{0} A Q_{0} Q_{1}$ has the desired normal form with regard to the basis $e_{1}, e_{2}, \cdots, e_{n-1}$ : and we have only to extend $P_{1}$ and $Q_{1}$ to the original basis, $e_{1}, e_{2}, \cdots, e_{n}$, by adding the conditions $P_{1} e_{n}=e_{n}=Q_{1} e_{n}$ in order to have $A$ in the required normal form with regard to this basis.

Assume now that the rank of $A$ is $n$. If the coefficients of $A$ have a H.C.F., $f(\lambda)$, we may write $A(\lambda)=f(\lambda) A_{1}(\lambda)$ : therefore, since multiplying $P(\lambda) A(\lambda) Q(\lambda)$ by a scalar factor still leaves it in the normal form, we may, without loss of generality, assume that there is no common factor.

If $x=\left(\xi_{1}, \xi_{2}, \cdots, \xi_{n}\right)$ is a constant vector, $A x$ can only vanish for values of $\lambda$ for which $|A|=0$ : but the values of $\lambda$ for which $A x=0$ are necessarily continuous functions of the $\xi$ 's and, since the coefficients of $A$ have no common factor in $R$, there are therefore no values of $\lambda$ in $R$ for which $A x=0$ for every constant vector $x$; hence there is some constant vector, $x$, for which $A x \neq 0$ for any value of $\lambda$ in $R$. Let $X$ be a constant matrix whose first column consists of the coördinates of this vector, the remaining coefficients being so chosen as to make the determinant of $X$ not zero. The coefficients in the first column of $A X$ are then the coefficients of $A x$ and therefore have no factor in common.

Let $A X e_{1}=\Sigma a_{r 1}(\lambda) e_{r}$. By Lemma III, we can find a matric function $P_{1}^{-1}(\lambda)$, holomorphic and non-singular in $R$, which is such that

$$
P_{1}^{-1} e_{1}=\sum a_{r 1}(\lambda) e_{r},
$$

whence $P_{1} A X e_{1}=e_{1}$. If, therefore, 


$$
P_{1} A X e_{r}=c_{r 1} e_{1}+\sum_{s=2}^{n} c_{r s} e_{s} \quad(r=2,3, \cdots, n),
$$

and $Q_{1}$ is the matrix defined by

$$
e_{1}=Q_{1} e_{1}, \quad e_{r}-c_{r 1} e_{1}=Q_{1} e_{r} \quad(r=2,3, \cdots, n),
$$

then $Q_{1}$ is a matric function, holomorphic in $R$, for which $\left|Q_{1}\right|=1$ and

$$
\begin{array}{r}
P_{1} A X Q_{1} e_{1}=e_{1}, \quad P_{1} A X Q_{1} e_{r}=P_{1} A X\left(e_{r}-c_{r 1} e_{1}\right)=\sum_{s=2}^{n} c_{r s} e_{s} \\
(r=2,3, \cdots, n) .
\end{array}
$$

All the coefficients in the first row and column of $P_{1} A X Q_{1}$ are therefore zero, except the first, which is 1 . Striking out this row and column, we have a matrix of order $n-1$, and, as we have assumed the theorem true for matrices of order less than $n$, this matrix can be reduced to the required form by means of two matric functions $P_{2}$ and $Q_{2}$ which are defined relative to the basis $e_{2}, e_{3}, \cdots, e_{n}$ and are holomorphic and non-singular in $R$. If we extend these matrices to the basis $e_{1}, e_{2}, \cdots, e_{n}$ as above by adding the conditions $P_{2} e_{1}=e_{1}=Q_{2} e_{1}$, the matrix $P_{2} P_{1} A X Q_{1} Q_{2}$ has the form required by the theorem since in multiplying by $P_{2}$ and $Q_{2}$ the first row and column of $P_{1} A X Q_{1}$ remain unaltered; the induction is therefore complete. 